Commun. Korean Math. Soc. 26 (2011), No. 1, pp. 51-65

DOI 10.4134/CKMS.2011.26.1.051

\title{
ITERATIVE METHODS FOR GENERALIZED EQUILIBRIUM PROBLEMS AND NONEXPANSIVE MAPPINGS
}

\author{
Sun Young Cho, Shin Min Kang, and XiaOlong Qin
}

\begin{abstract}
In this paper, a composite iterative process is introduced for a generalized equilibrium problem and a pair of nonexpansive mappings. It is proved that the sequence generated in the purposed composite iterative process converges strongly to a common element of the solution set of a generalized equilibrium problem and of the common fixed point of a pair of nonexpansive mappings.
\end{abstract}

\section{Introduction and preliminaries}

Throughout this paper, we always assume that $H$ is a real Hilbert space with inner product $\langle\cdot, \cdot\rangle$ and norm $\|\cdot\|$. Let $C$ be a nonempty closed convex subset of $H$ and $A: C \rightarrow H$ be a nonlinear mapping.

Recall the following definitions.

(1) $A$ is said to be monotone if

$$
\langle A x-A y, x-y\rangle \geq 0, \quad \forall x, y \in C .
$$

(2) $A$ is said to be strongly monotone if there exists a constant $\alpha>0$ such that

$$
\langle A x-A y, x-y\rangle \geq \alpha\|x-y\|^{2}, \quad \forall x, y \in C .
$$

For such a case, $T$ is also said to be $\alpha$-strongly-monotone.

(3) $A$ is said to be inverse-strongly monotone if there exists a constant $\alpha>0$ such that

$$
\langle A x-A y, x-y\rangle \geq \alpha\|A x-A y\|^{2}, \quad \forall x, y \in C .
$$

For such a case, $A$ is also said to be $\alpha$-inverse-strongly monotone.

Recall that the classical variational inequality problem, denoted by $V I(C, A)$, is to find $x \in C$ such that

$$
\langle A x, y-x\rangle \geq 0, \quad \forall y \in C .
$$

Received September 28, 2010.

2010 Mathematics Subject Classification. 47H05, 47H09, 47J05, 47J25.

Key words and phrases. equilibrium problem, nonexpansive mapping, inverse-strongly monotone mapping, contractive mapping. 
It is well known that $z \in H$ and $x \in C$ satisfy the inequality

$$
\langle x-z, y-x\rangle \geq 0, \quad \forall y \in C
$$

if and only if $x=P_{C} z$, where $P_{C}$ denotes the metric projection from $H$ onto $C$. From the above, we see that $x \in C$ is a solution to the problem (1.1) if and only if $x$ is a fixed point of the mapping $P_{C}(I-\rho A)$, where $\rho>0$ is a constant and $I$ is the identity mapping.

Let $A: C \rightarrow H$ be an $\alpha$-inverse-strongly monotone mapping and $F$ be a bifunction from $C \times C$ into $\mathbb{R}$, where $\mathbb{R}$ denotes the set of real numbers. We consider the following generalized equilibrium problem:

$$
\text { Find } x \in C \text { such that } F(x, y)+\langle A x, y-x\rangle \geq 0, \quad \forall y \in C .
$$

In this paper, the set of such an $x \in C$ is denoted by $\operatorname{EP}(F, A)$, i.e.,

$$
E P(F, A)=\{x \in C: F(x, y)+\langle A x, y-x\rangle \geq 0, \forall y \in C\} .
$$

Next, we give some special cases of the problem (1.2).

(I) If $A \equiv 0$, the zero mapping, then the generalized equilibrium problem (1.2) is reduced to the following equilibrium problem:

$$
\text { Find } x \in C \text { such that } F(x, y) \geq 0, \quad \forall y \in C \text {. }
$$

In this paper, the set of such an $x \in C$ is denoted by $E P(F)$, i.e.,

$$
E P(F)=\{x \in C: F(x, y) \geq 0, \forall y \in C\} .
$$

(II) If $F \equiv 0$, then the problem (1.2) is reduced to the classical variational inequality problem (1.1).

The problem (1.2) is very general in the sense that it includes, as special cases, optimization problems, variational inequalities, mini-max problems, the Nash equilibrium problem in noncooperative games and others; see, for instance, $[1,4,5,11]$.

Recently, many authors considered iterative method for the problems (1.1), (1.2) and (1.3); see, for example, $[1,3,4,5,6,7,8,9,10,11,13,14,15,17,18]$ for more details.

To study the equilibrium problems (1.2) and (1.3), we may assume that $F$ satisfies the following conditions:

(A1) $F(x, x)=0$ for all $x \in C$,

(A2) $F$ is monotone, i.e., $F(x, y)+F(y, x) \leq 0$ for all $x, y \in C$,

(A3) for each $x, y, z \in C$,

$$
\limsup _{t \downarrow 0} F(t z+(1-t) x, y) \leq F(x, y),
$$

(A4) for each $x \in C, y \mapsto F(x, y)$ is convex and weakly lower semi-continuous.

Let $T: C \rightarrow C$ be a nonlinear mapping. In this paper, we use $F(T)$ to denote the set of fixed points of $T$. Recall the following definitions. 
(4) The mapping $T$ is said to be contractive if there exists a constant $\lambda \in$ $(0,1)$ such that

$$
\|T x-T y\| \leq \lambda\|x-y\|, \quad \forall x, y \in C .
$$

(5) The mapping $T$ is said to be nonexpansive if

$$
\|T x-T y\| \leq\|x-y\|, \quad \forall x, y \in C .
$$

In 2005, H. Iiduka and W. Takahashi [9] considered the classical variational inequality (1.1) and a nonexpansive mapping. To be more precise, they obtained the following results.

Theorem IT. Let $C$ be a closed convex subset of a real Hilbert space $H$. Let $A$ be an $\alpha$-inverse-strongly monotone mapping from $C$ into $H$ and $T$ be a nonexpansive mapping from $C$ into itself such that $F(T) \cap V I(C, A) \neq \emptyset$. Suppose that $x_{1}=x \in C$ and $\left\{x_{n}\right\}$ is given by

$$
x_{n+1}=\alpha_{n} x+\left(1-\alpha_{n}\right) T P_{C}\left(x_{n}-\lambda_{n} A x_{n}\right), \quad \forall n \geq 1,
$$

where $\left\{\alpha_{n}\right\}$ is a sequence in $[0,1)$ and $\left\{\lambda_{n}\right\}$ is a sequence in $[0,2 \alpha]$. If $\left\{\alpha_{n}\right\}$ and $\left\{\lambda_{n}\right\}$ are chosen so that $\left\{\lambda_{n}\right\} \subset[a, b]$ for some $a, b$ with $0<a<b<2 \alpha$,

$$
\begin{aligned}
& \lim _{n \rightarrow \infty} \alpha_{n}=0, \quad \sum_{n=1}^{\infty} \alpha_{n}=\infty, \quad \sum_{n=1}^{\infty}\left|\alpha_{n+1}-\alpha_{n}\right|<\infty \quad \text { and } \\
& \sum_{n=1}^{\infty}\left|\lambda_{n+1}-\lambda_{n}\right|<\infty
\end{aligned}
$$

then $\left\{x_{n}\right\}$ converges strongly to $P_{F(T) \cap V I(C, A)} x$.

Recently, S. Takahashi and W. Takahashi [17] considered the equilibrium problem (1.3) by an iterative method. To be more precise, they proved the following theorem.

Theorem TT1. Let $C$ be a nonempty closed convex subset of a real Hilbert space $H$. Let $F$ be a bifunction from $C \times C$ into $\mathbb{R}$ satisfying (A1)-(A4) and $T$ be a nonexpansive mapping of $C$ into $H$ such that $F(T) \cap E P(F) \neq \emptyset$. Let $f$ be a contraction from $H$ into itself and let $\left\{x_{n}\right\}$ and $\left\{y_{n}\right\}$ be sequences generated by $x_{1} \in H$ and

$$
\left\{\begin{array}{l}
F\left(y_{n}, u\right)+\frac{1}{r_{n}}\left\langle u-y_{n}, y_{n}-x_{n}\right\rangle \geq 0, \quad \forall u \in C, \\
x_{n+1}=\alpha_{n} f\left(x_{n}\right)+\left(1-\alpha_{n}\right) T y_{n}, \quad \forall n \geq 1,
\end{array}\right.
$$

where $\left\{\alpha_{n}\right\} \in[0,1]$ and $\left\{r_{n}\right\} \subset(0, \infty)$ satisfy

$$
\begin{aligned}
& \lim _{n \rightarrow \infty} \alpha_{n}=0, \quad \sum_{n=1}^{\infty} \alpha_{n}=\infty, \quad \sum_{n=1}^{\infty}\left|\alpha_{n+1}-\alpha_{n}\right|<\infty, \\
& \liminf _{n \rightarrow \infty} r_{n}>0 \quad \text { and } \quad \sum_{n=1}^{\infty}\left|r_{n+1}-r_{n}\right|<\infty
\end{aligned}
$$


Then $\left\{x_{n}\right\}$ and $\left\{y_{n}\right\}$ converge strongly to $z \in F(T) \cap E P(F)$, where $z=$ $P_{F(T) \cap E P(F)} f(z)$.

Very recently, S. Takahashi and W. Takahashi [18] further considered the generalized equilibrium problem (1.2). They obtained the following result in a real Hilbert space.

Theorem TT2. Let $C$ be a closed convex subset of a real Hilbert space $H$ and $F: C \times C \rightarrow \mathbb{R}$ be a bifunction satisfying (A1)-(A4). Let $A$ be an $\alpha$-inversestrongly monotone mapping from $C$ into $H$ and $S$ be a non-expansive mapping from $C$ into itself such that $F(S) \cap E P(F, A) \neq \emptyset$. Let $u \in C$ and $x_{1} \in C$ and let $\left\{z_{n}\right\} \subset C$ and $\left\{x_{n}\right\} \subset C$ be sequences generated by

$$
\begin{gathered}
\qquad\left\{\begin{array}{l}
F\left(z_{n}, y\right)+\left\langle A x_{n}, y-z_{n}\right\rangle+\frac{1}{r_{n}}\left\langle y-z_{n}, z_{n}-x_{n}\right\rangle \geq 0, \quad \forall y \in C, \\
x_{n+1}=\beta_{n} x_{n}+\left(1-\beta_{n}\right) S\left[\alpha_{n} u+\left(1-\alpha_{n}\right) z_{n}\right], \quad \forall n \geq 1,
\end{array}\right. \\
\text { where }\left\{\alpha_{n}\right\} \subset[0,1],\left\{\beta_{n}\right\} \subset[0,1] \text { and }\left\{r_{n}\right\} \subset[0,2 \alpha] \text { satisfy } \\
0<c \leq \beta_{n} \leq d<1, \quad 0<a \leq \lambda_{n} \leq b<2 \alpha, \\
\lim _{n \rightarrow \infty}\left|\lambda_{n}-\lambda_{n+1}\right|=0, \quad \lim _{n \rightarrow \infty} \alpha_{n}=0 \quad \text { and } \quad \sum_{n=1}^{\infty} \alpha_{n}=\infty .
\end{gathered}
$$

Then $\left\{x_{n}\right\}$ converges strongly to $z=P_{F(S) \cap E P(F, A)} u$.

In this paper, motivated by the research going on in this direction, we introduce a composite iterative algorithm for the problem of finding a common element in the solution set of the generalized equilibrium problem (1.2) and in the common fixed point set of a pair of nonexpansive mappings. Strong convergence theorems are established in the framework of Hilbert spaces. The results presented in this paper improve and extend the corresponding results announced by H. Iiduka and W. Takahashi [9] and S. Takahashi and W. Takahashi $[17,18]$ and some others.

In order to prove our main results, we also need the following definitions and lemmas.

Recall that a Hilbert space $H$ is said to satisfy Opial's condition [12] if, for any sequence $\left\{x_{n}\right\} \subset H$ with $x_{n} \rightarrow x$, then the inequality

$$
\liminf _{n \rightarrow \infty}\left\|x_{n}-x\right\|<\liminf _{n \rightarrow \infty}\left\|x_{n}-y\right\|
$$

holds for any $y \in H$ such that $y \neq x$.

The following lemma can be find in $[1,8]$.

Lemma 1.1. Let $C$ be a nonempty closed convex subset of a real Hilbert space $H$ and $F: C \times C \rightarrow \mathbb{R}$ be a bifunction satisfying(A1)-(A4). Then for any $r>0$ and $x \in H$, there exists $z \in C$ such that

$$
F(z, y)+\frac{1}{r}\langle y-z, z-x\rangle \geq 0, \quad \forall y \in C .
$$


Further, define

$$
T_{r} x=\left\{z \in C: F(z, y)+\frac{1}{r}\langle y-z, z-x\rangle \geq 0, \forall y \in C\right\}
$$

for all $r>0$ and $x \in H$. Then the following hold:

(a) $T_{r}$ is single-valued,

(b) $T_{r}$ is firmly nonexpansive, i.e., for any $x, y \in H$,

$$
\left\|T_{r} x-T_{r} y\right\|^{2} \leq\left\langle T_{r} x-T_{r} y, x-y\right\rangle
$$

(c) $F\left(T_{r}\right)=E P(F)$,

(d) $\left\|T_{s} x-T_{r} x\right\|^{2} \leq \frac{s-r}{s}\left\langle T_{s} x-T_{r} x, T_{s} x-x\right\rangle$,

(e) $\operatorname{EP}(F)$ is closed and convex.

Lemma $1.2([16])$. Let $\left\{x_{n}\right\}$ and $\left\{y_{n}\right\}$ be bounded sequences in a Banach space $E$ and let $\left\{\beta_{n}\right\}$ be a sequence in $[0,1]$ with

$$
0<\liminf _{n \rightarrow \infty} \beta_{n} \leq \limsup _{n \rightarrow \infty} \beta_{n}<1 .
$$

Suppose that $x_{n+1}=\left(1-\beta_{n}\right) y_{n}+\beta_{n} x_{n}$ for all integers $n \geq 0$ and

$$
\limsup _{n \rightarrow \infty}\left(\left\|y_{n+1}-y_{n}\right\|-\left\|x_{n+1}-x_{n}\right\|\right) \leq 0 \text {. }
$$

Then $\lim _{n \rightarrow \infty}\left\|y_{n}-x_{n}\right\|=0$.

Lemma 1.3 ([19]). Assume that $\left\{\alpha_{n}\right\}$ is a sequence of nonnegative real numbers such that

$$
\alpha_{n+1} \leq\left(1-\gamma_{n}\right) \alpha_{n}+\delta_{n}
$$

where $\left\{\gamma_{n}\right\}$ is a sequence in $(0,1)$ and $\left\{\delta_{n}\right\}$ is a sequence such that

(a) $\sum_{n=1}^{\infty} \gamma_{n}=\infty$,

(b) $\limsup _{n \rightarrow \infty} \frac{\delta_{n}}{\gamma_{n}} \leq 0$ or $\sum_{n=1}^{\infty}\left|\delta_{n}\right|<\infty$.

Then $\lim _{n \rightarrow \infty} \alpha_{n}=0$.

\section{Main results}

Now, we are ready to give our main results.

Theorem 2.1. Let $C$ be a nonempty closed convex subset of a real Hilbert space $H, F: C \times C \rightarrow \mathbb{R}$ be a bifunction satisfying (A1)-(A4) and $A: C \rightarrow H$ be an $\alpha$-inverse-strongly monotone mapping. Let $S$ and $T: C \rightarrow C$ be nonexpansive mappings such that $\mathcal{F}:=F(S) \cap F(T) \cap E P(F, A) \neq \emptyset$ and $f: C \rightarrow C$ be a contractive mapping with the coefficient $\lambda \in(0,1)$. Let $\left\{x_{n}\right\}$ be a sequence defined by the following algorithm: $x_{1} \in C$ and

$$
\left\{\begin{array}{l}
F\left(w_{n}, y\right)+\left\langle A x_{n}, y-w_{n}\right\rangle+\frac{1}{r_{n}}\left\langle y-w_{n}, w_{n}-x_{n}\right\rangle \geq 0, \quad \forall y \in C, \\
z_{n}=\gamma_{n} w_{n}+\left(1-\gamma_{n}\right) T w_{n}, \\
y_{n}=\alpha_{n} f\left(x_{n}\right)+\left(1-\alpha_{n}\right) z_{n}, \\
x_{n+1}=\beta_{n} x_{n}+\left(1-\beta_{n}\right) S y_{n}, \quad \forall n \geq 1
\end{array}\right.
$$


where $\left\{r_{n}\right\}$ is a positive sequence and $\left\{\alpha_{n}\right\},\left\{\beta_{n}\right\}$ and $\left\{\gamma_{n}\right\}$ are sequences in $[0,1]$. Assume that the following conditions are satisfied
(a) $0<c \leq \beta_{n} \leq d<1$,
(b) $0<a \leq r_{n} \leq b<2 \alpha$ and $\lim _{n \rightarrow \infty}\left(r_{n}-r_{n+1}\right)=0$,
(c) $\lim _{n \rightarrow \infty} \alpha_{n}=0$ and $\sum_{n=1}^{\infty} \alpha_{n}=\infty$,
(d) $\lim _{n \rightarrow \infty} \gamma_{n}=1$.

Then $\left\{x_{n}\right\}$ converges strongly to $q \in \mathcal{F}$, where $q=P_{\mathcal{F}} f(q)$.

Proof. Note that $w_{n}$ can be rewritten as $w_{n}=T_{r_{n}}\left(x_{n}-r_{n} A x_{n}\right)$ for each $n \geq 1$. Take $p \in \mathcal{F}$. Since $p=T_{r_{n}}\left(p-r_{n} A p\right)$ and $A$ is $\alpha$-inverse-strongly monotone and $0<r_{n}<2 \alpha$, we see that

$$
\begin{aligned}
\left\|w_{n}-p\right\|^{2} & =\left\|T_{r_{n}}\left(x_{n}-r_{n} A x_{n}\right)-T_{r_{n}}\left(p-r_{n} A p\right)\right\|^{2} \\
& \leq\left\|\left(x_{n}-r_{n} A x_{n}\right)-\left(p-r_{n} A p\right)\right\|^{2} \\
& =\left\|\left(x_{n}-p\right)-r_{n}\left(A x_{n}-A p\right)\right\|^{2} \\
& =\left\|x_{n}-p\right\|^{2}-2 r_{n}\left\langle x_{n}-p, A x_{n}-A p\right\rangle+r_{n}^{2}\left\|A x_{n}-A p\right\|^{2} \\
& \leq\left\|x_{n}-p\right\|^{2}-r_{n}\left(2 \alpha-r_{n}\right)\left\|A x_{n}-A p\right\|^{2} \\
& \leq\left\|x_{n}-p\right\|^{2} .
\end{aligned}
$$

Note that

$$
\begin{aligned}
\left\|z_{n}-p\right\| & \leq \gamma_{n}\left\|w_{n}-p\right\|+\left(1-\gamma_{n}\right)\left\|T w_{n}-p\right\| \\
& \leq \gamma_{n}\left\|w_{n}-p\right\|+\left(1-\gamma_{n}\right)\left\|w_{n}-p\right\| \\
& =\left\|w_{n}-p\right\| .
\end{aligned}
$$

On the other hand, we have

$$
\begin{aligned}
\left\|y_{n}-p\right\| & =\left\|\alpha_{n}\left(f\left(x_{n}\right)-p\right)+\left(1-\alpha_{n}\right)\left(z_{n}-p\right)\right\| \\
& \leq \alpha_{n} \lambda\left\|x_{n}-p\right\|+\alpha_{n}\|f(p)-p\|+\left(1-\alpha_{n}\right)\left\|x_{n}-p\right\| \\
& =\left[1-\alpha_{n}(1-\lambda)\right]\left\|x_{n}-p\right\|+\alpha_{n}\|f(p)-p\| .
\end{aligned}
$$

It follows that

$$
\begin{aligned}
\left\|x_{n+1}-p\right\| & \leq \beta_{n}\left\|x_{n}-p\right\|+\left(1-\beta_{n}\right)\left\|S y_{n}-p\right\| \\
& \leq \beta_{n}\left\|x_{n}-p\right\|+\left(1-\beta_{n}\right)\left\|y_{n}-p\right\| \\
& \leq\left[1-\alpha_{n}(1-\lambda)\left(1-\beta_{n}\right)\right]\left\|x_{n}-p\right\|+\alpha_{n}\left(1-\beta_{n}\right)\|f(p)-p\| .
\end{aligned}
$$

Putting $M_{1}=\max \left\{\left\|x_{1}-p\right\|, \frac{\|f(p)-p\|}{1-\lambda}\right\}$, we have that $\left\|x_{n}-p\right\| \leq M_{1}$ for all $n \geq 1$. Indeed, it is obvious that $\left\|x_{1}-p\right\| \leq M_{1}$. Suppose that $\left\|x_{k}-p\right\| \leq M_{1}$ for some positive integer $k$. Then we have that

$$
\begin{aligned}
\left\|x_{k+1}-p\right\| & \leq\left[1-\alpha_{k}(1-\lambda)\left(1-\beta_{k}\right)\right]\left\|x_{k}-p\right\|+\alpha_{k}\left(1-\beta_{k}\right)\|f(p)-p\| \\
& \leq\left[1-\alpha_{k}(1-\lambda)\left(1-\beta_{k}\right)\right] M_{1}+\alpha_{k}\left(1-\beta_{k}\right)(1-\lambda) M_{1} \\
& =M_{1} .
\end{aligned}
$$


This shows that $\left\|x_{n}-p\right\| \leq M_{1}$ for all $n \geq 1$. Hence $\left\{x_{n}\right\}$ is bounded. In view of Lemma 1.1, we see that

$$
\begin{aligned}
& \left\|w_{n+1}-w_{n}\right\| \\
\leq & \left\|T_{r_{n+1}}\left(I-r_{n+1} A\right) x_{n+1}-T_{r_{n+1}}\left(I-r_{n} A\right) x_{n}\right\| \\
& +\left\|T_{r_{n+1}}\left(I-r_{n} A\right) x_{n}-T_{r_{n}}\left(I-r_{n} A\right) x_{n}\right\| \\
\leq & \left\|\left(I-r_{n+1} A\right) x_{n+1}-\left(I-r_{n} A\right) x_{n}\right\| \\
& +\left\|T_{r_{n+1}}\left(I-r_{n} A\right) x_{n}-T_{r_{n}}\left(I-r_{n} A\right) x_{n}\right\| \\
\leq & \left\|x_{n+1}-x_{n}\right\|+\left|r_{n+1}-r_{n}\right|\left\|A x_{n}\right\| \\
& +\frac{\left|r_{n+1}-r_{n}\right|}{r_{n+1}}\left\|T_{r_{n+1}}\left(I-r_{n} A\right) x_{n}-\left(I-r_{n} A\right) x_{n}\right\| \\
\leq & \left\|x_{n+1}-x_{n}\right\|+\left|r_{n+1}-r_{n}\right|\left\|A x_{n}\right\|+\frac{\left|r_{n+1}-r_{n}\right|}{r_{n+1}} M_{2},
\end{aligned}
$$

where $M_{2}$ is an appropriate constant such that

$$
M_{2}=\sup \left\{\left\|T_{r_{n+1}}\left(I-r_{n} A\right) x_{n}-\left(I-r_{n} A\right) x_{n}\right\|: n \geq 1\right\} .
$$

Note that

$$
\begin{aligned}
\left\|z_{n+1}-z_{n}\right\| \leq & \gamma_{n+1}\left\|w_{n+1}-w_{n}\right\|+\left(1-\gamma_{n+1}\right) \mid\left\|T w_{n+1}-T w_{n}\right\| \\
& +\left|\gamma_{n+1}-\gamma_{n}\right|\left(\left\|w_{n}\right\|+\left\|T w_{n}\right\|\right) \\
\leq & \left\|w_{n+1}-w_{n}\right\|+\left|\gamma_{n+1}-\gamma_{n}\right| M_{3}
\end{aligned}
$$

where $M_{3}$ is an appropriate constant such that $M_{3}=\sup \left\{\left\|w_{n}\right\|+\left\|T w_{n}\right\|: n \geq\right.$ $1\}$. Substituting (2.3) into (2.4), we obtain that

$$
\begin{aligned}
\left\|z_{n+1}-z_{n}\right\| \leq & \left\|x_{n+1}-x_{n}\right\|+\left|r_{n+1}-r_{n}\right||| A x_{n} \|+\frac{\left|r_{n+1}-r_{n}\right|}{r_{n+1}} M_{2} \\
& +\left|\gamma_{n+1}-\gamma_{n}\right| M_{3} \\
\leq & \left\|x_{n+1}-x_{n}\right\|+\left(2\left|r_{n+1}-r_{n}\right|+\left|\gamma_{n+1}-\gamma_{n}\right|\right) M_{4},
\end{aligned}
$$

where $M_{4}$ is an appropriate constant such that

$$
M_{4}=\max \left\{\sup \left\{\left\|A x_{n}\right\|: n \geq 1\right\}, \frac{M_{2}}{a}, M_{3}\right\} .
$$

On the other hand, we have

$$
\begin{aligned}
\left\|y_{n+1}-y_{n}\right\| \leq & \alpha_{n+1}\left\|f\left(x_{n+1}\right)-f\left(x_{n}\right)\right\|+\left(1-\alpha_{n+1}\right)\left\|z_{n+1}-z_{n}\right\| \\
& +\left|\alpha_{n+1}-\alpha_{n}\right|\left\|f\left(x_{n}\right)-z_{n}\right\| \\
\leq & \lambda \alpha_{n+1}\left\|x_{n+1}-x_{n}\right\|+\left(1-\alpha_{n+1}\right) \mid\left\|z_{n+1}-z_{n}\right\| \\
& +\left|\alpha_{n+1}-\alpha_{n}\right|\left\|f\left(x_{n}\right)-z_{n}\right\| .
\end{aligned}
$$

Substituting (2.5) into (2.6), we obtain that

$$
\begin{aligned}
& \left\|y_{n+1}-y_{n}\right\| \\
\leq & \left\|x_{n+1}-x_{n}\right\|+\left(2\left|r_{n+1}-r_{n}\right|+\left|\gamma_{n+1}-\gamma_{n}\right|+\left|\alpha_{n+1}-\alpha_{n}\right|\right) M_{5},
\end{aligned}
$$


where $M_{5}$ is an appropriate constant such that $M_{5}=\max \left\{\sup \left\{\left\|f\left(x_{n}\right)-z_{n}\right\|\right.\right.$ : $\left.n \geq 1\}, M_{4}\right\}$. It follows from (2.7) that

$$
\begin{aligned}
& \left\|S y_{n+1}-S y_{n}\right\| \\
\leq & \left\|y_{n+1}-y_{n}\right\| \\
\leq & \left\|x_{n+1}-x_{n}\right\|+\left(2\left|r_{n+1}-r_{n}\right|+\left|\gamma_{n+1}-\gamma_{n}\right|+\left|\alpha_{n+1}-\alpha_{n}\right|\right) M_{5} .
\end{aligned}
$$

This in turn implies that

$$
\begin{aligned}
& \left\|S y_{n+1}-S y_{n}\right\|-\left\|x_{n+1}-x_{n}\right\| \\
\leq & \left(2\left|r_{n+1}-r_{n}\right|+\left|\gamma_{n+1}-\gamma_{n}\right|+\left|\alpha_{n+1}-\alpha_{n}\right|\right) M_{5} .
\end{aligned}
$$

From the conditions (b)-(d), we see that

$$
\limsup _{n \rightarrow \infty}\left(\left\|S y_{n+1}-S y_{n}\right\|-\left\|x_{n+1}-x_{n}\right\|\right) \leq 0 .
$$

In view of Lemma 1.2 , we see that

$$
\lim _{n \rightarrow \infty}\left\|S y_{n}-x_{n}\right\|=0 .
$$

Consequently, we obtain that

$$
\lim _{n \rightarrow \infty}\left\|x_{n+1}-x_{n}\right\|=\lim _{n \rightarrow \infty}\left(1-\beta_{n}\right)\left\|S y_{n}-x_{n}\right\|=0 .
$$

It follows from (2.2) that

$$
\begin{aligned}
& \left\|x_{n+1}-p\right\|^{2} \\
\leq & \beta_{n}\left\|x_{n}-p\right\|^{2}+\left(1-\beta_{n}\right)\left\|S y_{n}-p\right\|^{2} \\
\leq & \beta_{n}\left\|x_{n}-p\right\|^{2}+\left(1-\beta_{n}\right)\left(\left\|\alpha_{n}\left(f\left(x_{n}\right)-p\right)+\left(1-\alpha_{n}\right)\left(z_{n}-p\right)\right\|^{2}\right) \\
\leq & \beta_{n}\left\|x_{n}-p\right\|^{2}+\alpha_{n}\left\|f\left(x_{n}\right)-p\right\|^{2}+\left(1-\beta_{n}\right)\left\|z_{n}-p\right\|^{2} \\
\leq & \beta_{n}\left\|x_{n}-p\right\|^{2}+\alpha_{n}\left\|f\left(x_{n}\right)-p\right\|^{2}+\left(1-\beta_{n}\right)\left\|w_{n}-p\right\|^{2} .
\end{aligned}
$$

In view (2.1), we see that

$$
\begin{aligned}
& \left\|x_{n+1}-p\right\|^{2} \\
\leq & \left\|x_{n}-p\right\|^{2}+\alpha_{n}\left\|f\left(x_{n}\right)-p\right\|^{2}-r_{n}\left(1-\beta_{n}\right)\left(2 \alpha-r_{n}\right)\left\|A x_{n}-A p\right\|^{2} .
\end{aligned}
$$

From the conditions (a) and (b), we arrive at

$$
\begin{aligned}
& a(1-d)(2 \alpha-b)\left\|A x_{n}-A p\right\|^{2} \\
\leq & \left\|x_{n}-p\right\|^{2}-\left\|x_{n+1}-p\right\|^{2}+\alpha_{n}\left\|f\left(x_{n}\right)-p\right\|^{2} \\
\leq & \left(\left\|x_{n}-p\right\|+\left\|x_{n+1}-p\right\|\right)\left\|x_{n}-x_{n+1}\right\|+\alpha_{n}\left\|f\left(x_{n}\right)-p\right\|^{2} .
\end{aligned}
$$

It follows from (2.9) and the condition (c) that

$$
\lim _{n \rightarrow \infty}\left\|A x_{n}-A p\right\|=0 .
$$


On the other hand, we have

$$
\begin{aligned}
& \left\|w_{n}-p\right\|^{2} \\
= & \left\|T_{r_{n}}\left(I-r_{n} A\right) x_{n}-T_{r_{n}}\left(I-r_{n} A\right) p\right\|^{2} \\
\leq & \left\langle\left(I-r_{n} A\right) x_{n}-\left(I-r_{n} A\right) p, w_{n}-p\right\rangle \\
= & \frac{1}{2}\left(\left\|\left(I-r_{n} A\right) x_{n}-\left(I-r_{n} A\right) p\right\|^{2}+\left\|w_{n}-p\right\|^{2}\right. \\
& \left.-\left\|\left(I-r_{n} A\right) x_{n}-\left(I-r_{n} A\right) p-\left(w_{n}-p\right)\right\|^{2}\right) \\
= & \frac{1}{2}\left(\left\|x_{n}-p\right\|^{2}+\left\|w_{n}-p\right\|^{2}-\left\|x_{n}-w_{n}-r_{n}\left(A x_{n}-A p\right)\right\|^{2}\right) \\
= & \frac{1}{2}\left[\left\|x_{n}-p\right\|^{2}+\left\|w_{n}-p\right\|^{2}-\left(\left\|x_{n}-w_{n}\right\|^{2}\right.\right. \\
& \left.\left.-2 r_{n}\left\langle x_{n}-w_{n}, A x_{n}-A p\right\rangle+r_{n}^{2}\left\|A x_{n}-A p\right\|^{2}\right)\right] .
\end{aligned}
$$

This implies that

(2.12) $\quad\left\|w_{n}-p\right\|^{2} \leq\left\|x_{n}-p\right\|^{2}-\left\|x_{n}-w_{n}\right\|^{2}+2 r_{n}\left\|x_{n}-w_{n}\right\|\left\|A x_{n}-A p\right\|$.

Combining (2.10) with (2.12), we arrive at

$$
\begin{aligned}
\left\|x_{n+1}-p\right\|^{2} \leq & \left\|x_{n}-p\right\|^{2}+\alpha_{n}\left\|f\left(x_{n}\right)-p\right\|^{2}-\left(1-\beta_{n}\right)\left\|x_{n}-w_{n}\right\|^{2} \\
& +2 r_{n}\left\|x_{n}-w_{n}\right\|\left\|A x_{n}-A p\right\| .
\end{aligned}
$$

From the condition (a), we see that

$$
\begin{aligned}
& (1-d)\left\|x_{n}-w_{n}\right\|^{2} \\
\leq & \left\|x_{n}-p\right\|^{2}-\left\|x_{n+1}-p\right\|^{2}+\alpha_{n}\left\|f\left(x_{n}\right)-p\right\|^{2} \\
& +2 r_{n}\left\|x_{n}-w_{n}\right\|\left\|A x_{n}-A p\right\| \\
\leq & \left(\left\|x_{n}-p\right\|+\left\|x_{n+1}-p\right\|\right)\left\|x_{n}-x_{n+1}\right\|+\alpha_{n}\left\|f\left(x_{n}\right)-p\right\|^{2} \\
& +2 r_{n}\left\|x_{n}-w_{n}\right\|\left\|A x_{n}-A p\right\| .
\end{aligned}
$$

In view of the condition (c), (2.9) and (2.11), we obtain that

$$
\lim _{n \rightarrow \infty}\left\|x_{n}-w_{n}\right\|=0 \text {. }
$$

Note that

$$
\left\|z_{n}-w_{n}\right\| \leq\left(1-\gamma_{n}\right)\left\|T w_{n}-w_{n}\right\| .
$$

From the condition $(\mathrm{d})$, we obtain that

$$
\lim _{n \rightarrow \infty}\left\|z_{n}-w_{n}\right\|=0
$$

On the other hand, we have

$$
\begin{aligned}
\left\|S y_{n}-y_{n}\right\| & \leq\left\|S y_{n}-x_{n}\right\|+\left\|x_{n}-w_{n}\right\|+\left\|w_{n}-y_{n}\right\| \\
& \leq\left\|S y_{n}-x_{n}\right\|+\left\|x_{n}-w_{n}\right\|+\left\|w_{n}-z_{n}\right\|+\left\|z_{n}-y_{n}\right\| \\
& \leq\left\|S y_{n}-x_{n}\right\|+\left\|x_{n}-w_{n}\right\|+\left\|w_{n}-z_{n}\right\|+\alpha_{n}\left\|f\left(x_{n}\right)-z_{n}\right\| .
\end{aligned}
$$


In view of the condition (c), (2.8), (2.13) and (2.14), we see that

$$
\lim _{n \rightarrow \infty}\left\|S y_{n}-y_{n}\right\|=0 \text {. }
$$

Note that $P_{\mathcal{F}} f$ is a contractive mapping. It follows that $P_{\mathcal{F}} f$ has a unique fixed point. Next, we use $q$ to denote the unique fixed point. Now, we claim that

$$
\limsup _{n \rightarrow \infty}\left\langle f(q)-q, y_{n}-q\right\rangle \leq 0 .
$$

To show this inequality, take a subsequence $\left\{y_{n_{i}}\right\}$ of $\left\{y_{n}\right\}$ such that

$$
\limsup _{n \rightarrow \infty}\left\langle f(q)-q, y_{n}-q\right\rangle=\lim _{i \rightarrow \infty}\left\langle f(q)-q, y_{n_{i}}-q\right\rangle .
$$

We may, without loss of generality, assume that $y_{n_{i}} \rightarrow \eta$. Since $C$ is closed and convex, we see that $C$ is weakly closed. So, we have $\eta \in C$. Next, we show that $\eta \in \mathcal{F}$. First, we show $\eta \in E P(F, A)$. Note that

$$
\begin{aligned}
\left\|y_{n}-w_{n}\right\| & \leq\left\|y_{n}-z_{n}\right\|+\left\|z_{n}-w_{n}\right\| \\
& \leq \alpha_{n}\left\|f\left(x_{n}\right)-z_{n}\right\|+\left\|z_{n}-w_{n}\right\| .
\end{aligned}
$$

In view of the condition (c) and (2.14), we obtain that $\lim _{n \rightarrow \infty}\left\|y_{n}-w_{n}\right\|=0$. It follows that $w_{n_{i}} \rightarrow \eta$. Since $w_{n}=T_{r_{n}}\left(x_{n}-r_{n} A x_{n}\right)$, for any $y \in C$, we have

$$
F\left(w_{n}, y\right)+\left\langle A x_{n}, y-w_{n}\right\rangle+\frac{1}{r_{n}}\left\langle y-w_{n}, w_{n}-x_{n}\right\rangle \geq 0 .
$$

From the condition (A2), we see

$$
\left\langle A x_{n}, y-w_{n}\right\rangle+\frac{1}{r_{n}}\left\langle y-w_{n}, w_{n}-x_{n}\right\rangle \geq F\left(y, w_{n}\right) .
$$

Replacing $n$ by $n_{i}$, we arrive at

$$
\left\langle A x_{n_{i}}, y-w_{n_{i}}\right\rangle+\left\langle y-w_{n_{i}}, \frac{w_{n_{i}}-x_{n_{i}}}{r_{n_{i}}}\right\rangle \geq F\left(y, w_{n_{i}}\right) .
$$

For $t$ with $0<t \leq 1$ and $y \in C$, let $y_{t}=t y+(1-t) \eta$. Since $y \in C$ and $\eta \in C$, we have $y_{t} \in C$. It follows from (2.17) that

$$
\begin{aligned}
& \left\langle y_{t}-w_{n_{i}}, A y_{t}\right\rangle \\
\geq & \left\langle y_{t}-w_{n_{i}}, A y_{t}\right\rangle-\left\langle A x_{n_{i}}, y_{t}-w_{n_{i}}\right\rangle-\left\langle y_{t}-w_{n_{i}}, \frac{w_{n_{i}}-x_{n_{i}}}{r_{n_{i}}}\right\rangle \\
& +F\left(y_{t}, w_{n_{i}}\right) \\
= & \left\langle y_{t}-w_{n_{i}}, A y_{t}-A w_{n_{i}}\right\rangle+\left\langle y-w_{n_{i}}, A w_{n_{i}}-A x_{n_{i}}\right\rangle \\
& -\left\langle y_{t}-w_{n_{i}}, \frac{w_{n_{i}}-x_{n_{i}}}{r_{n_{i}}}\right\rangle+F\left(y_{t}, w_{n_{i}}\right) .
\end{aligned}
$$

From (2.13), we have $A w_{n_{i}}-A x_{n_{i}} \rightarrow 0$ as $i \rightarrow \infty$. On the other hand, from the monotonicity of $A$, we obtain that

$$
\left\langle y_{t}-w_{n_{i}}, A y_{t}-A w_{n_{i}}\right\rangle \geq 0 .
$$


It follows from (A4) and (2.18) that

$$
\left\langle y_{t}-\eta, A y_{t}\right\rangle \geq F\left(y_{t}, \eta\right) .
$$

From (A1), (A4) and (2.19), we see

$$
\begin{aligned}
0 & =F\left(y_{t}, y_{t}\right) \leq t F\left(y_{t}, y\right)+(1-t) F\left(y_{t}, \eta\right) \\
& \leq t F\left(y_{t}, y\right)+(1-t)\left\langle y_{t}-\eta, A y_{t}\right\rangle \\
& =t F\left(y_{t}, y\right)+(1-t) t\left\langle y-\eta, A y_{t}\right\rangle,
\end{aligned}
$$

which yields that

$$
F\left(y_{t}, y\right)+(1-t)\left\langle y-\eta, A y_{t}\right\rangle \geq 0 .
$$

Letting $t \rightarrow 0$ in the above inequality, we arrive at

$$
F(\eta, y)+\langle y-\eta, A \eta\rangle \geq 0 .
$$

This shows that $\eta \in E P(F, A)$. Next, we show that $\eta \in F(S)$. If not, we have $\eta \neq S \eta$. In view of the Opial condition, we see that

$$
\begin{aligned}
\liminf _{i \rightarrow \infty}\left\|y_{n_{i}}-\eta\right\| & <\liminf _{i \rightarrow \infty}\left\|y_{n_{i}}-S \eta\right\| \\
& \leq \liminf _{i \rightarrow \infty}\left\{\left\|y_{n_{i}}-S y_{n_{i}}\right\|+\left\|S y_{n_{i}}-S \eta\right\|\right\} \\
& \leq \liminf _{i \rightarrow \infty}\left\{\left\|y_{n_{i}}-S y_{n_{i}}\right\|+\left\|y_{n_{i}}-\eta\right\|\right\} .
\end{aligned}
$$

It follows that

$$
\liminf _{i \rightarrow \infty}\left\|y_{n_{i}}-\eta\right\|<\liminf _{i \rightarrow \infty}\left\|y_{n_{i}}-\eta\right\|
$$

which derives a contradiction. Thus, we have $\eta \in F(S)$. Next, we show that $\eta \in$ $F(T)$. If not, we have $\eta \neq T \eta$. Define a mapping $R_{n}$ by $R_{n} x=\gamma_{n} x+\left(1-\gamma_{n}\right) T x$ for each $x \in C$. It follows that $R_{n}$ is nonexpansive and $F(T)=F\left(R_{n}\right)$ for each $n \geq 1$. On the other hand, we from (2.14) know that $\left\|R_{n} w_{n}-w_{n}\right\| \rightarrow 0$ as $n \rightarrow \infty$. From the Opial condition, we see that

$$
\begin{aligned}
\liminf _{i \rightarrow \infty}\left\|w_{n_{i}}-\eta\right\| & <\liminf _{i \rightarrow \infty}\left\|w_{n_{i}}-R_{n_{i}} \eta\right\| \\
& \leq \liminf _{i \rightarrow \infty}\left\{\left\|w_{n_{i}}-R_{n_{i}} w_{n_{i}}\right\|+\left\|R_{n_{i}} w_{n_{i}}-R_{n_{i}} \eta\right\|\right\} \\
& \leq \liminf _{i \rightarrow \infty}\left\{\left\|w_{n_{i}}-R_{n_{i}} w_{n_{i}}\right\|+\left\|w_{n_{i}}-\eta\right\|\right\} .
\end{aligned}
$$

This also derives a contradiction. This implies that $\eta \in F\left(R_{n}\right)=F(T)$ for each $n \geq 1$. This shows that $\eta \in \mathcal{F}$. From (2.16), we see that

$$
\limsup _{n \rightarrow \infty}\left\langle f(q)-q, y_{n}-q\right\rangle=\langle f(q)-q, \eta-q\rangle \leq 0 .
$$

Finally, we show that $x_{n} \rightarrow q$ as $n \rightarrow \infty$. Note that

$$
\begin{aligned}
& \left\|x_{n+1}-q\right\|^{2} \\
= & \beta_{n}\left\langle x_{n}-q, x_{n+1}-q\right\rangle+\left(1-\beta_{n}\right)\left\langle S y_{n}-q, x_{n+1}-q\right\rangle \\
\leq & \beta_{n}\left\|x_{n}-q\right\|\left\|x_{n+1}-q\right\|+\left(1-\beta_{n}\right)\left\|S y_{n}-q\right\|\left\|x_{n+1}-q\right\| \\
\leq & \frac{\beta_{n}}{2}\left(\left\|x_{n}-q\right\|^{2}+\left\|x_{n+1}-q\right\|^{2}\right)+\frac{1-\beta_{n}}{2}\left(\left\|y_{n}-q\right\|^{2}+\left\|x_{n+1}-q\right\|^{2}\right) .
\end{aligned}
$$


It follows that

$$
\left\|x_{n+1}-q\right\|^{2} \leq \beta_{n}\left\|x_{n}-q\right\|^{2}+\left(1-\beta_{n}\right)\left\|y_{n}-q\right\|^{2} .
$$

On the other hand, we have

$$
\begin{aligned}
\left\|y_{n}-q\right\|^{2}= & \alpha_{n}\left\langle f\left(x_{n}\right)-q, y_{n}-q\right\rangle+\left(1-\alpha_{n}\right)\left\langle z_{n}-q, y_{n}-q\right\rangle \\
\leq & \alpha_{n}\left\langle f\left(x_{n}\right)-q, y_{n}-q\right\rangle+\left(1-\alpha_{n}\right)\left\|z_{n}-q\right\|\left\|y_{n}-q\right\| \\
\leq & \alpha_{n}\left\langle f\left(x_{n}\right)-f(q), y_{n}-q\right\rangle+\alpha_{n}\left\langle f(q)-q, y_{n}-q\right\rangle \\
& +\left(1-\alpha_{n}\right)\left\|x_{n}-q\right\|\left\|y_{n}-q\right\| \\
\leq & {\left[1-\alpha_{n}(1-\lambda)\right]\left\|x_{n}-q\right\|\left\|y_{n}-q\right\|+\alpha_{n}\left\langle f(q)-q, y_{n}-q\right\rangle } \\
\leq & \frac{1-\alpha_{n}(1-\lambda)}{2}\left(\left\|x_{n}-q\right\|^{2}+\left\|y_{n}-q\right\|^{2}\right)+\alpha_{n}\left\langle f(q)-q, y_{n}-q\right\rangle .
\end{aligned}
$$

It follows that

$$
\left\|y_{n}-q\right\|^{2} \leq\left[1-\alpha_{n}(1-\lambda)\right]\left\|x_{n}-q\right\|^{2}+2 \alpha_{n}\left\langle f(q)-q, y_{n}-q\right\rangle .
$$

Substituting (2.22) into (2.21), we arrive at

$$
\begin{aligned}
\left\|x_{n+1}-q\right\|^{2} \leq & {\left[1-\alpha_{n}\left(1-\beta_{n}\right)(1-\lambda)\right]\left\|x_{n}-q\right\|^{2} } \\
& +2 \alpha_{n}\left(1-\beta_{n}\right)\left\langle f(q)-q, y_{n}-q\right\rangle .
\end{aligned}
$$

In view of (2.16), we from Lemma 1.3 see that $x_{n} \rightarrow q$ as $n \rightarrow \infty$. This completes the proof.

Remark 2.2. Theorem 2.1 includes Theorem TT2 as a special case. To be more precise, if $f(x)=u \in C$ for each $x \in C$ and $\gamma_{n}=1$ for each $n \geq 1$, then Theorem 2.1 is reduced to Theorem TT2.

From Theorem 2.1, we have the following results on the equilibrium problem (1.3).

Corollary 2.3. Let $C$ be a nonempty closed convex subset of a real Hilbert space $H$ and $F: C \times C \rightarrow \mathbb{R}$ be a bifunction satisfying (A1)-(A4). Let $S: C \rightarrow C$ be a nonexpansive mapping such that $\mathcal{F}:=F(S) \cap E P(F) \neq \emptyset$ and $f: C \rightarrow C$ be a contractive mapping with the coefficient $\lambda \in(0,1)$. Let $\left\{x_{n}\right\}$ be a sequence defined by the following algorithm: $x_{1} \in C$ and

$$
\left\{\begin{array}{l}
F\left(w_{n}, y\right)+\frac{1}{r_{n}}\left\langle y-w_{n}, w_{n}-x_{n}\right\rangle \geq 0, \quad \forall y \in C, \\
y_{n}=\alpha_{n} f\left(x_{n}\right)+\left(1-\alpha_{n}\right) w_{n}, \\
x_{n+1}=\beta_{n} x_{n}+\left(1-\beta_{n}\right) S y_{n}, \quad \forall n \geq 1,
\end{array}\right.
$$

where $\left\{r_{n}\right\}$ is a positive sequence and $\left\{\alpha_{n}\right\},\left\{\beta_{n}\right\}$ and $\left\{\gamma_{n}\right\}$ are sequences in $[0,1]$. Assume that the following conditions are satisfied

(a) $0<c \leq \beta_{n} \leq d<1$,

(b) $0<a \leq r_{n} \leq b<2 \alpha$ and $\lim _{n \rightarrow \infty}\left(r_{n}-r_{n+1}\right)=0$,

(c) $\lim _{n \rightarrow \infty} \alpha_{n}=0$ and $\sum_{n=1}^{\infty} \alpha_{n}=\infty$.

Then $\left\{x_{n}\right\}$ converges strongly to $q \in \mathcal{F}$, where $q=P_{\mathcal{F}} f(q)$. 
Proof. Putting $A=0$ in Theorem 2.1, we can obtain the desired conclusion immediately.

Corollary 2.4. Let $C$ be a nonempty closed convex subset of a real Hilbert space $H$ and $A: C \rightarrow H$ be an $\alpha$-inverse-strongly monotone mapping. Let $S$ and $T$ : $C \rightarrow C$ be nonexpansive mappings such that $\mathcal{F}:=F(S) \cap F(T) \cap V I(C, A) \neq \emptyset$ and $f: C \rightarrow C$ be a contractive mapping with the coefficient $\lambda \in(0,1)$. Let $\left\{x_{n}\right\}$ be a sequence defined by the following algorithm: $x_{1} \in C$ and

$$
\left\{\begin{array}{l}
z_{n}=\gamma_{n} P_{C}\left(x_{n}-r_{n} A x_{n}\right)+\left(1-\gamma_{n}\right) T P_{C}\left(x_{n}-r_{n} A x_{n}\right) \\
y_{n}=\alpha_{n} f\left(x_{n}\right)+\left(1-\alpha_{n}\right) z_{n}, \\
x_{n+1}=\beta_{n} x_{n}+\left(1-\beta_{n}\right) S y_{n}, \quad \forall n \geq 1
\end{array}\right.
$$

where $\left\{r_{n}\right\}$ is a positive sequence and $\left\{\alpha_{n}\right\},\left\{\beta_{n}\right\}$ and $\left\{\gamma_{n}\right\}$ are sequences in $[0,1]$. Assume that the following conditions are satisfied
(a) $0<c \leq \beta_{n} \leq d<1$,
(b) $0<a \leq r_{n} \leq b<2 \alpha$ and $\lim _{n \rightarrow \infty}\left(r_{n}-r_{n+1}\right)=0$,
(c) $\lim _{n \rightarrow \infty} \alpha_{n}=0$ and $\sum_{n=1}^{\infty} \alpha_{n}=\infty$,
(d) $\lim _{n \rightarrow \infty} \gamma_{n}=1$.

Then $\left\{x_{n}\right\}$ converges strongly to $q \in \mathcal{F}$, where $q=P_{\mathcal{F}} f(q)$.

Proof. In Theorem 2.1, putting $F(x, y)=0$ for each $x, y \in C$, we see that

$$
\left\langle A x_{n}, y-w_{n}\right\rangle+\frac{1}{r_{n}}\left\langle y-w_{n}, w_{n}-x_{n}\right\rangle \geq 0, \quad \forall y \in C
$$

is equivalent to

$$
\left\langle x_{n}-r_{n} A x_{n}-w_{n}, w_{n}-y\right\rangle \geq 0, \quad \forall y \in C .
$$

This implies that $w_{n}=P_{C}\left(x_{n}-r_{n} A x_{n}\right)$. From Theorem 2.1, we can obtain the desired results immediately.

Recall that $T: C \rightarrow C$ is said to be a $k$-strictly pseudocontractive [2] if there exists a constant $k \in[0,1)$ such that

$$
\|T x-T y\|^{2} \leq\|x-y\|^{2}+k\|(I-T) x-(I-T) y\|^{2}, \quad \forall x, y \in C .
$$

From [2], we see that if $T$ is $k$-strictly pseudocontractive, then $I-T$ is $\frac{1-k}{2}$ inverse-strongly monotone.

The following theorem is not hard to derive.

Corollary 2.5. Let $C$ be a nonempty closed convex subset of a real Hilbert space $H, F: C \times C \rightarrow \mathbb{R}$ be a bifunction satisfying (A1)-(A4) and $T_{A}: C \rightarrow C$ be an $k$-strictly pseudocontractive mapping. Let $S$ and $T: C \rightarrow C$ be nonexpansive mappings such that $\mathcal{F}:=F(S) \cap F(T) \cap E P\left(F, I-T_{A}\right) \neq \emptyset$ and $f: C \rightarrow C$ be a contractive mapping with the coefficient $\lambda \in(0,1)$. Let $\left\{x_{n}\right\}$ be a sequence 
defined by the following algorithm: $x_{1} \in C$ and

$$
\left\{\begin{array}{l}
F\left(w_{n}, y\right)+\left\langle x_{n}-T_{A} x_{n}, y-w_{n}\right\rangle+\frac{1}{r_{n}}\left\langle y-w_{n}, w_{n}-x_{n}\right\rangle \geq 0, \quad \forall y \in C, \\
z_{n}=\gamma_{n} w_{n}+\left(1-\gamma_{n}\right) T w_{n}, \\
y_{n}=\alpha_{n} f\left(x_{n}\right)+\left(1-\alpha_{n}\right) z_{n}, \\
x_{n+1}=\beta_{n} x_{n}+\left(1-\beta_{n}\right) S y_{n}, \quad \forall n \geq 1,
\end{array}\right.
$$

where $\left\{r_{n}\right\}$ is a positive sequence and $\left\{\alpha_{n}\right\},\left\{\beta_{n}\right\}$ and $\left\{\gamma_{n}\right\}$ are sequences in $[0,1]$. Assume that the following conditions are satisfied

(a) $0<c \leq \beta_{n} \leq d<1$,

(b) $0<a \leq r_{n} \leq b<1-k$ and $\lim _{n \rightarrow \infty}\left(r_{n}-r_{n+1}\right)=0$,

(c) $\lim _{n \rightarrow \infty} \alpha_{n}=0$ and $\sum_{n=1}^{\infty} \alpha_{n}=\infty$,

(d) $\lim _{n \rightarrow \infty} \gamma_{n}=1$.

Then $\left\{x_{n}\right\}$ converges strongly to $q \in \mathcal{F}$, where $q=P_{\mathcal{F}} f(q)$.

\section{References}

[1] E. Blum and W. Oettli, From optimization and variational inequalities to equilibrium problems, Math. Student 63 (1994), no. 1-4, 123-145.

[2] F. E. Browder and W. V. Petryshyn, Construction of fixed points of nonlinear mappings in Hilbert space, J. Math. Anal. Appl. 20 (1967), 197-228.

[3] L. C. Ceng and J. C. Yao, Hybrid viscosity approximation schemes for equilibrium problems and fixed point problems of infinitely many nonexpansive mappings, Appl. Math. Comput. 198 (2008), no. 2, 729-741.

[4] O. Chadli, N. C. Wong and J. C. Yao, Equilibrium problems with applications to eigenvalue problems, J. Optim. Theory Appl. 117 (2003), no. 2, 245-266.

[5] S. S. Chang, H. W. Joseph Lee and C. K. Chan, A new method for solving equilibrium problem fixed point problem and variational inequality problem with application to optimization, Nonlinear Anal. 70 (2009), no. 9, 3307-3319.

[6] J. Chen, L. Zhang and T. Fan, Viscosity approximation methods for nonexpansive mappings and monotone mappings, J. Math. Anal. Appl. 334 (2007), no. 2, 1450-1461.

[7] V. Colao, G. Marino and H. K. Xu, An iterative method for finding common solutions of equilibrium and fixed point problems, J. Math. Anal. Appl. 344 (2008), no. 1, 340-352.

[8] P. L. Combettes and S. A. Hirstoaga, Equilibrium programming in Hilbert spaces, J. Nonlinear Convex Anal. 6 (2005), no. 1, 117-136.

[9] H. Iiduka and W. Takahashi, Strong convergence theorems for nonexpansive mappings and inverse-strongly monotone mappings, Nonlinear Anal. 61 (2005), no. 3, 341-350.

[10] S. M. Kang, S. Y. Cho and Z. Liu, Convergence of iterative sequences for generalized equilibrium problems involving inverse-strongly monotone mappings, J. Inequal. Appl. 2010 (2010), Article ID 827082, 16 pages.

[11] A. Moudafi and M. Thera, Proximal and dynamical approaches to equilibrium problems, Ill-posed variational problems and regularization techniques (Trier, 1998), 187-201, Lecture Notes in Econom. and Math. Systems, 477, Springer, Berlin, 1999.

[12] Z. Opial, Weak convergence of the sequence of successive approximation for nonexpansive mappings, Bull. Amer. Math. Soc. 73 (1967), 561-597.

[13] S. Plubtieng and R. Punpaeng, A new iterative method for equilibrium problems and fixed point problems of nonexpansive mappings and monotone mappings, Appl. Math. Comput. 197 (2008), no. 2, 548-558.

[14] X. Qin, Y. J. Cho and S. M. Kang, Convergence theorems of common elements for equilibrium problems and fixed point problems in Banach spaces, J. Comput. Appl. Math. 225 (2009), no. 1, 20-30. 
[15] X. Qin, M. Shang and Y. Su, Strong convergence of a general iterative algorithm for equilibrium problems and variational inequality problems, Math. Comput. Modelling 48 (2008), no. 7-8, 1033-1046.

[16] T. Suzuki, Strong convergence of Krasnoselskii and Mann's type sequences for oneparameter nonexpansive semigroups without Bochner integrals, J. Math. Anal. Appl. 305 (2005), no.1, 227-239.

[17] S. Takahashi and W. Takahashi, Viscosity approximation methods for equilibrium problems and fixed point problems in Hilbert spaces, J. Math. Anal. Appl. 331 (2007), no. $1,506-515$.

[18] _ Strong convergence theorem for a generalized equilibrium problem and a nonexpansive mapping in a Hilbert space, Nonlinear Anal. 69 (2008), no. 3, 1025-1033.

[19] H. K. Xu, Iterative algorithms for nonlinear operators, J. London Math. Soc. (2) 66 (2002), no. 1, 240-256.

Sun Young Cho

Department of Mathematics

Gyeongsang National University

JINJU 660-701, KoREA

E-mail address: ooly61@yahoo.co.kr

SHIN Min KAng

Department of Mathematics AND RINS

Gyeongsang National University

JiNJU 660-701, KOREA

E-mail address: smkang@gnu.ac.kr

XiAOLONG QIN

Department of Mathematics

HANGZHOU NORMAL UNIVERSITY

Hangzhou 310036, P. R. China

E-mail address: 1jjhqxl@yahoo.com.cn; qxlxajh@163.com 